\title{
Hematopoietic Adverse Events Associated with BNT162b2 mRNA Covid-19 Vaccine
}

\author{
Batuhan ERDOGDU, Olgu Erkin CINAR, Umit Yavuz MALKAN, Salih AKSU, \\ Haluk DEMIROGLU, Yahya BUYUKASIK, Hakan GOKER, Nilgun SAYINALP, \\ Ibrahim Celalettin HAZNEDAROGLU
}

Hacettepe University, Faculty of Medicine, Department of Hematology, Ankara, TURKEY

To the Editor,

Chimerism-mediated immunotherapy by means of vaccination at the individual and community level seems to be the only choice to end the currently ongoing COVID-19 pandemic. ${ }^{1}$ The BNT162b2 mRNA COVID-19 vaccine has shown a safety and efficacy profile against COVID-19 amongst immunocompetent individuals. A two-dose regimen of $\mathrm{BNT} 162 \mathrm{~b} 2$ provided $95 \%$ protection against COVID-19 in people of age $\geq 16$ years old. Although vaccination is necessary for hematological patients, due to the low antibody response in this population, an adequate response cannot be obtained with vaccination. Atay and coworkers brilliantly disclosed the clinical course of COVID-19 in patients complicated with hematological disorders in UHOD. ${ }^{2} \mathrm{We}$, herein with this case-series, would like to report hematopoietic adverse events due to the administration of the BNT162b2 mRNA COVID-19 vaccine presenting with distinct clinical presentations.

Our first case is a 22-year-old female patient who had no known disease, following the second day of BNT162b2 mRNA COVID-19 vaccine, found to be neutropenia (neutrophil/leucocyte 400/1400/ $\mu \mathrm{L}$ ) hemoglobin and thrombocyte was found within normal range, peripheral smear is similar complete blood count, blastic or other atypical cells was not found. The patient was followed up in terms of moderate neutropenia. After seven days, the patient's neutrophil/leucocyte was 1000/2900/ $\mathrm{LL}$.
Our second case is a 49-year-old female patient, two days after receiving BNT162b2 mRNA COVID-19 vaccine, petechiae was started in arms and then in lower extremities. The complaints of weakness and fatigue continued to increase within days. The patient, who was admitted to the hospital ten days later with these complaints, was found to have bi-cytopenia. Bone marrow aspiration and biopsy reported that $20-30 \%$ stained with cd19 diffuse positive Terminal deoxynucleotidyl transferase (TdT) in blastic cells. HyperCVAD chemotherapy was started with the diagnosis of b-acute lymphoblastic leukemia (B-ALL).

Our third case is a 57-year-old male patient, difficulty in swallowing, and oral ulceration after vaccination of BNT162b2 mRNA COVID-19 vaccine, found to be neutropenic (neutrophil/leucocyte $200 / 1000 / \mu \mathrm{L}$ ) and other parameters were found within normal range. Peripheral blood smear was reported as normocytic erythroid morphology, tear cells in a few areas, marked neutropenia, platelet count consistent with complete blood count, blast cells not observed. After seven days, neutropenia continued and so a bone marrow biopsy was performed for the etiology of cytopenia. It came as normocellular bone marrow, 5\% blastic cells and mild megaloblastic/dysplastic changes in the erythroid series. 
Our fourth case is a 47-year-old female patient, diagnosed with B-cell lymphoma 2 years ago, underwent autologous stem cell transplantation after 8 cycles of R-EPOCH chemotherapy. While she was being followed up in remission at the 14th month, she applied for dyspnea and fatigue that started after the BNT162b2 mRNA COVID-19 vaccine. Bicytopenia and blasts were seen, and diagnosed acute lymphoblastic leukemia. The treatment of the patient with a septic course continues in the intensive care unit. Our last case is a 67-year-old female patient who was diagnosed with acute myeloid leukemia in 2007. The patient, who was in remission after chemotherapy, underwent an allogeneic hematopoietic stem cell transplant in 2008 from full-matched donor. The patient, who was followed up in remission for 14 years after the transplant, had normal blood follow-ups in the controls after two doses of inactivated SARS-CoV-2 vaccine in 07/2021. After the BNT162b2 mRNA COVID-19 vaccine, fatigue and weakness started in 09/2021, the tests were repeated and pancytopenia was detected. The bone marrow was infiltrated with $90 \%$ blasts, acute myeloid leukemia was diagnosed and venetoclax-azacitidine treatment was started.

On the basis of previous studies related to COVID-19, the hematological changes like leukopenia, lymphopenia, thrombocytopenia and coagulopathy were shown. ${ }^{3}$ It can be thought these adverse effects occurred by way of SARS-CoV-2 spike protein. In the literature, there are many studies on the relationship between SARS-CoV-2 spike protein and cellular changes of hematopoietic cells. Ropa et al. first found that SARS-CoV-2 spike protein induce responses in hematopoietic stem cells (HSC), hematopoietic progenitors (HP) by the way of ACE2 receptor, which is primarily targeted receptor for SARS-CoV-2. The obtained data showed a significantly $33 \%$ and $38 \%$ decrease in CD34+ cells, granulocyte-monocyte progenitors (GMP) respectively with SARS-CoV-2 spiked protein exposure. Also, peripheral blood monocytes displayed morphological changes like smaller and more granular. This study suggested that exposure to SARS$\mathrm{CoV}-2$ spike protein impacts hematopoiesis and myeloid differentiation in vitro and ex vivo. ${ }^{4}$

Our research team first presented the concept that there occurs a local hematopoietic RAS (renin-an- giotensin system) in the bone marrow (BM) about two decades ago. Local BM RAS has important outcomes on hematopoietic systems, especially on myeloid and erythroid cells. ${ }^{5}$ It has been proven in previous studies that there are important receptors of Ang 1-7 and Mas in the bone marrow stroma. ${ }^{6}$ $\mathrm{BM}$ stromal microenvironment includes AT1R and AT2R (angiotensin type 1 and type 2 receptors, respectively) and inhibitory tetrapeptide AcSDKP (N-acetyl-Ser-Asp-Lys-Pro). The major RAS effector agent angiotensin II (Ang II) exerts its effects on the hematopoietic system by activating the AT1Rs and AT2Rs, along with the BM microenvironment. The reason behind this targeting is entering into cells as a result of causing alteration to the normal functions of ACE2. The ACE2 receptor enables $\mathrm{S}$ proteins to be activated and the stimulated $\mathrm{S}$ proteins support the SARS-CoV-2 virus to enter the host cells1. It can be observed that ACE2 receptor and SARS-CoV-2 entry-facilitating transmembrane protease TMPRSS2 are expressed by all types of stem cells studied in a research by Kucia et al. Furthermore, the researchers determined activation of Nlrp3 inflammasomes in response to viral spike protein. This activation was inhibited by exposure of the stimulated cells to angiotensin 1-7.7 Hui et al. demonstrated that the SARS-CoV-2 spike protein significantly inhibits DNA damage repair by hindering key DNA repair protein 53BP1 on the basis of an in vitro cell culture study. Also, it can be observed that the spike protein localizes in the nucleus. The obtained data showed that the SARS-CoV-2 full-length spike protein inhibits DNA damage repair by impeding DNA repair protein recruitment. ${ }^{8}$ The full-length spike protein present in BNT162b2 vaccine may be related to the inhibition of DNA damage repair. Severe SARS-Cov-2/COVID-19 infection may lead to the damage of hematopoietic stem/progenitor cell and endothelial progenitor cells since COVID-19 infection frequently presented with anemia, lymphopenia, and thrombocytopenia. This negative effect of the virus on human hematopoiesis and endothelium has been shown in infected patients and demonstrated in vitro after exposure of cells to SARS-Cov-2/COVID-19 spike protein. ${ }^{9}$ SARS-Cov-2 entry receptor ACE2 is expressed on very small CD45-precursors of hematopoietic and endothelial cells and the interaction of the ACE2 
receptor with the SARS-CoV-2 spike protein activates the Nlrp3 inflammasome. Activated Nlrp3 inflammasome induces cell death by pyroptosis and over-activation of Nlrp3 inflammasome could trigger the cytokine "storm" and may damage hematopoietic stem cells. ${ }^{10}$

In conclusion, all of this literature data favors that SARS-Cov-2 affects hematopoiesis and may lead to cytopenias. Viral spike protein cytotoxicity exerts damage to hematopoietic stem cells. On the other hand, the mRNA vaccines produce endogenous spike protein. All over the world, during the ongoing pandemic, millions of people have already got vaccinated with the novel mRNA technology; however hematological side effects occur only in a minority of them. This fact leads us to consider that the endogenous spike protein which is produced after the mRNA vaccine administration could be eliminated with reactive compensatory immune mechanisms, which prevents to occur adverse hematopoietic effects of the vaccines in the bone marrow microenvironment. In the future, there is a need for randomized studies in order to clarify which subpopulation is susceptible to the mRNA vaccine-associated hematopoietic adverse events and the micro-environmental mechanisms beneath these side effects.

\section{REFERENCES}

1. Turk S, Turk C, Malkan UY, et al. Current community transmission and future perspectives on the COVID-19 process. Turk J Med Sci 51: 1001-1011, 2021.

2. Atay MH, Okuyucu M, Gullu YT, et al. Clinical course of Covid-19 in hematological disorders. UHOD-Int J Hematol Oncol 31: 153-160, 2021.

3. Frater JL, Zini G, d'Onofrio G, Rogers HJ. COVID-19 and the clinical hematology laboratory. Int J Lab Hematol 42 Suppl 1 : 11-18, 2020.

4. Ropa J, Cooper S, Capitano ML, Broxmeyer HE. Sars-Cov-2 Spike Protein Induces Cellular Changes in primitive and mature hematopoietic cells. Blood 136: 25-26, 2020.

5. Haznedaroglu IC, Beyazit Y. Local bone marrow renin-angiotensin system in primitive, definitive and neoplastic haematopoiesis. Clin Sci (Lond) 124: 307-323, 2013.

6. Heringer-Walther S, Eckert K, Schumacher SM, et al. Angiotensin-(1-7) stimulates hematopoietic progenitor cells in vitro and in vivo. Haematologica 94: 857-860, 2009.
7. Ratajczak MZ, Bujko K, Ciechanowicz A, et al. SARS-CoV-2 entry receptor ACE2 is expressed on very small CD45precursors of hematopoietic and endothelial cells and in response to virus spike protein activates the Nirp3 inflammasome. Stem Cell Reviews and Reports 17: 266-277, 2021.

8. Jiang H, Mei YF. SARS-CoV-2 Spike Impairs DNA damage repair and inhibits $V(D) J$ recombination in vitro. Viruses 13: 2056-2066, 2021.

9. Ropa J, Cooper S, Capitano ML, et al. Human hematopoietic stem, progenitor, and immune cells respond Ex Vivo to SARS-CoV-2 Spike Protein. Stem Cell Rev Rep 17: 253-265, 2021.

10. Ratajczak MZ, Kucia M. SARS-CoV-2 infection and overactivation of Nlrp3 inflammasome as a trigger of cytokine "storm" and risk factor for damage of hematopoietic stem cells. Leukemia. 34: 1726-1729, 2020.

\section{Correspondence:}

\section{Dr. Batuhan ERDOGDU}

Hacettepe Universitesi, Tip Fakultesi

Hematoloji Anabilim Dali

Sihhiye, ANKARA / TURKEY

Tel: (+90-553) 5995362

e-mail: dr.batuhan@gmail.com

\section{ORCIDs:}

$\begin{array}{ll}\text { Batuhan Erdogdu } & 0000-0001-8968-3917 \\ \text { Olgu Erkin Cinar } & 0000-0003-1226-5797 \\ \text { Umit Yavuz Malkan } & 0000-0001-5444-4895 \\ \text { Salih Aksu } & 0000-0003-0634-3808 \\ \text { Haluk Demiroglu } & 0000-0002-6790-8748 \\ \text { Yahya Buyukasik } & 0000-0002-4764-2348 \\ \text { Hakan Göker } & 0000-0002-1039-7756 \\ \text { Nilgün Sayinalp } & 0000-0002-4782-896 x \\ \text { lbrahim Celalettin Haznedaroglu } & 0000-0001-8028-9462\end{array}$

